\title{
Clinical trials using molecular stratification of pediatric brain tumors
}

\author{
Samuel Z. Hanz ${ }^{1}$, Oluwaseyi Adeuyan ${ }^{1}$, Grace Lieberman ${ }^{2}$, Tammy Hennika ${ }^{2}$ \\ ${ }^{1}$ Department of Neurological Surgery, ${ }^{2}$ Department of Pediatrics, Division of Child Neurology, Weill Cornell Medicine, New York, NY, USA \\ Contributions: (I) Conception and design: SZ Hanz, T Hennika; (II) Administrative support: None; (III) Provision of study materials or patients: \\ None; (IV) Collection and assembly of data: SZ Hanz, O Adeuyan, G Lieberman; (V) Data analysis and interpretation: SZ Hanz, T Hennika; (VI) \\ Manuscript writing: All authors; (VII) Final approval of manuscript: All authors. \\ Correspondence to: Tammy Hennika, MD. Department of Pediatrics, Division of Child Neurology, Weill Cornell Medicine, 525 East 68th Street, Box \\ 91, New York, NY 10065, USA. Email: hennikatammy@gmail.com.
}

\begin{abstract}
Brain cancer is now the leading cause of cancer death in children and adolescents, surpassing leukemia. The heterogeneity and invasiveness of pediatric brain tumors have historically made them difficult to treat. Although surgical intervention and standard of care therapies such as radiation and chemotherapy have improved the outlook for those affected, results are often transient and lend themselves to tumor recurrence or resistance. There also still exists a subset of brain tumors which remain unresponsive to treatment altogether. Therefore, there is great need for new therapeutic approaches. With the recent advent of molecularly-driven technologies, many of these complex tumors can now be classified by integrating molecular profiling data with clinical information such as demographics and outcomes. This new knowledge has allowed for the molecular stratification of pediatric brain tumors into distinct subgroups and the identification of molecular targets, which is changing how these children are treated, namely in the setting of clinical trials. Notable examples include reduced doses of radiation and chemotherapy in the winglessactivated subgroup of medulloblastoma, which has a favorable prognosis, and novel experimental drugs targeting BRAF alterations in low-grade gliomas and dopamine receptors in high-grade gliomas. In this review, we highlight several key previous and ongoing clinical trials that utilize molecular stratifications and targets for the treatment of pediatric brain tumors.
\end{abstract}

Keywords: Brain tumor; clinical trials; molecular; pediatric

Submitted Dec 03, 2019. Accepted for publication Feb 26, 2020.

doi: $10.21037 /$ tp. 2020.03 .04

View this article at: http://dx.doi.org/10.21037/tp.2020.03.04

\section{Introduction}

Pediatric brain cancer has replaced leukemia as the leading type of cancer causing death among children and adolescents aged 1-19 years (1). For those children that survive, many will have long-term side effects from radiation and chemotherapy treatments, such as impaired motor function, neuropathy, endocrine dysfunction, neurocognitive deficits, as well as psychosocial late effects (2). Therefore, there is a great need for novel treatments to improve patient outcomes and reduce long-term side effects. Although pediatric brain tumors have traditionally been classified by histologic grade, recent genomic studies have made significant contributions to our knowledge of key molecular drivers in these tumors. As a result, the 2016 World Health Organization (WHO) revision integrated histology with molecular features in the classification of central nervous system tumors (3). In addition to important diagnostic information, this newly acquired molecular profiling data provides significant prognostic value. A prominent example is the embryonal brain tumor medulloblastoma $(\mathrm{MB})$ which has been reliably stratified into distinct molecular subgroups based on patient demographics, clinical presentations and outcomes, and genetic alterations [wingless (WNT)-activated, sonic 
Table 1 Molecular targeted clinical trials in pediatric gliomas

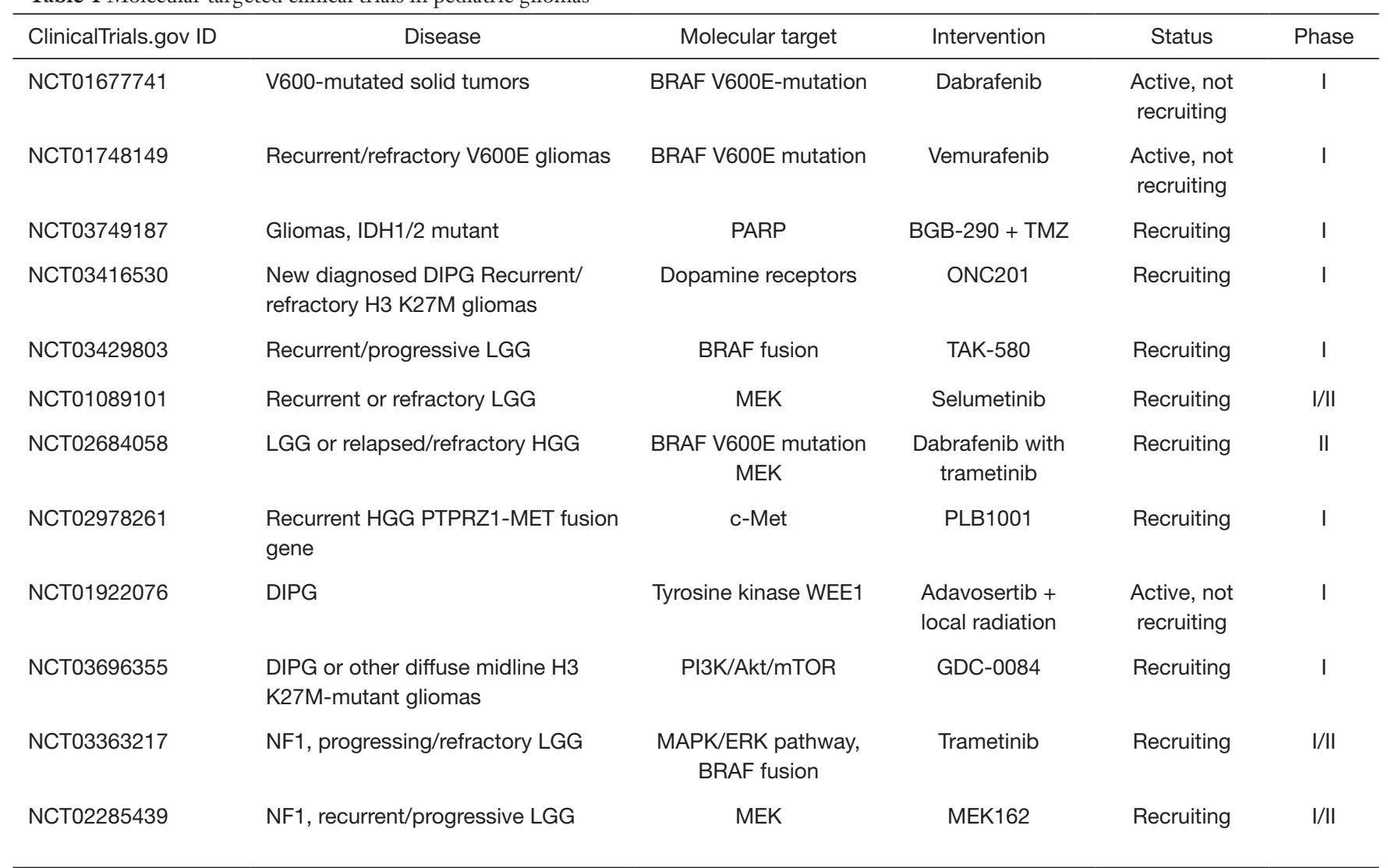

TMZ, temozolomide; LGG, low-grade glioma; HGG, high-grade glioma; DIPG, diffuse intrinsic pontine glioma; PI3K, phosphoinositide 3-kinase; IDH, isocitrate dehydrogenase; mTOR, mammalian target of rapamycin; Akt, protein kinase B.

hedgehog $(\mathrm{SHH})$-activated and tumor suppressor protein p53 (TP53)-mutant, SHH-activated and TP53-wildtype, and non-WNT/non-SHH (group 3 and group 4)], wherein group $3 \mathrm{MBs}$ carry the worst prognosis of all the subgroups and TP53 mutation status is the most important risk factor in SHH-activated MB (4-6). Similarly, pediatric low-grade gliomas (PLGGs) harbor different anomalies in the BRAF gene (B-Raf proto-oncogene or $\mathrm{v}$-Raf murine sarcoma viral oncogene homolog B1) which offers information on the tumor phenotype as well as the patient's overall survival $(7,8)$. Furthermore, those with pediatric high-grade gliomas (PHGGs) exhibit different prognoses based on mutations in genes encoding the histone variants $\mathrm{H} 3.3$ (H3F3A) and H3.1 (HIST1H3B) (9). Given the clinical significance of these molecular differences, recent clinical trials aim to identify driver mutations and other biomarkers to allow for targeted therapy. This review presents the recent literature and highlights active clinical trials that utilize the molecular stratification of pediatric brain tumors and specific tumor molecular targets for novel therapeutic strategies. A focus is made on molecular targeted pediatric clinical trials involving low grade gliomas (LGGs) and high grade gliomas (HGGs) (Table 1), MBs (Table 2), and those which encompass multiple brain tumor types (Table 3).

\section{PLGG}

LGGs are the most frequent brain tumors in children (10). The overall survival is excellent, reaching 90 percent when the tumors are completely resected. However, tumors that have low potential for resection, such as optic pathway and brainstem gliomas, can be difficult to treat (11). The WHO classifies PLGGs as grade I or grade II representative of a heterogeneous set of tumors (3). Recent studies have identified alterations in the BRAF gene in PLGGs. 
Table 2 Molecular targeted clinical trials in pediatric medulloblastoma

\begin{tabular}{|c|c|c|c|c|c|}
\hline ClinicalTrials.gov ID & Disease & Molecular target & Intervention & Status & Phase \\
\hline NCT01878617 & $\begin{array}{l}\text { Newly diagnosed MB (WNT, } \\
\text { SHH, non-WNT, non-SHH) }\end{array}$ & Smoothened & $\begin{array}{l}\text { Vismodegib, craniospinal } \\
\text { irradiation, chemotherapy }\end{array}$ & Recruiting & II \\
\hline NCT02724579 & Newly diagnosed WNT MB & WNT-driven & $\begin{array}{l}\text { Reduced craniospinal } \\
\text { irradiation and chemotherapy }\end{array}$ & Recruiting & II \\
\hline
\end{tabular}

SHH, sonic hedgehog; MB, medulloblastoma; CK2, casein kinase 2; WNT, wingless; chk1, checkpoint kinase 1.

Table 3 Molecular targeted clinical trials encompassing multiple pediatric brain tumors

\begin{tabular}{lllll}
\hline ClinicalTrials.gov ID & \multicolumn{1}{c}{ Disease } & \multicolumn{1}{c}{ Molecular target } & \multicolumn{1}{c}{ Intervention } & Status \\
\hline NCT03434262 (SJDAWN) & Recurrent brain tumors & MEK, CDK4/6, smoothened & $\begin{array}{l}\text { Trametinib, ribociclib, } \\
\text { sonidegib, gemcitabine }\end{array}$ & Recruiting \\
NCl-COG pediatric MATCH & $\begin{array}{l}\text { Multiple refractory/ } \\
\text { recurrent pediatric } \\
\text { cancers }\end{array}$ & $\begin{array}{l}\text { MEK, BRAF (and multiple } \\
\text { other targets) }\end{array}$ & $\begin{array}{l}\text { Selumetinib, vemurafenib } \\
\text { (and multiple other } \\
\text { drugs) }\end{array}$ \\
\hline
\end{tabular}

BRAF encodes the serine/threonine kinase B-Raf, a downstream effector in the mitogen-activated protein kinase (MAPK) signaling pathway and is considered to be the most important kinase in the MAPK (Ras/Raf/MEK/ ERK) cascade reactions $(7,12,13)$. Recent research has attributed activation of the MAPK signaling pathway in the tumorigenesis of PLGGs (14). Two common BRAF alterations have been implicated in the etiology of these tumors: KIAA1549-BRAF fusions and $\mathrm{BRAF}^{\mathrm{V} 600 \mathrm{E}}$ mutations. Several studies between 2008 and 2009 uncovered the first event, a tandem duplication in chromosome $7 \mathrm{q} 34$ which produced a fusion gene between BRAF and the previously unidentified KIAA1549 (15). Not only did the KIAA1549BRAF fusion result in constitutive activation of the MAPK pathway, a study by Lin et al. [2012] revealed that the fusion was overexpressed relative to wild-type (12). Given the proportion of pilocytic astrocytomas (WHO grade I) that harbor a BRAF fusion $(\sim 70 \%)$, this aberration has become a diagnostic hallmark for these tumors. The $\mathrm{BRAF}^{\mathrm{V} 600 \mathrm{E}}$ mutation is a point mutation in BRAF resulting in an amino acid substitution from valine to glutamic acid at position $600(16,17)$. This mutation is found at much lower frequencies in pilocytic astrocytomas, diffuse gangliogliomas, and pilomyxoid astrocytomas, but is highly prevalent in pleomorphic xanthoastrocytomas (70-80\%) followed by gangliogliomas $(20-50 \%)(8,18)$. Although both the KIAA1549-BRAF fusion and BRAF ${ }^{\mathrm{V} 600 \mathrm{E}}$ mutation exhibit oncogenic potential by constitutively activating the MAPK pathway, they produce histologically distinct tumors which also confer different patient outcomes. PLGGs with the $\mathrm{BRAF}^{\mathrm{V} 600 \mathrm{E}}$ mutation are not as heterogeneous as PLGGs with KIAA1549-BRAF fusions, yet patients with $\mathrm{BRAF}^{\mathrm{V} 600 \mathrm{E}}$ mutation exhibit a higher risk for progression and overall shorter survival $(7,8)$. These differences suggest that the genetic events may not be operating in the same fashion and thus should be targeted separately.

Children with neurofibromatosis type 1 (NF1) and tuberous sclerosis complex (TSC), two genetic neurocutaneous syndromes, are predisposed to develop PLGGs. NF1 is caused by a mutation in the NF1 tumor-suppressor gene which codes for a large protein, neurofibromin, that negatively regulates the RAS/MAPK pathway. The loss of neurofibromin increases RAS activity, resulting in hyperactive signaling of both the MAPK and the phosphatidylinositol-3-kinase ( $\mathrm{PI} 3 \mathrm{~K})$ - protein kinase B (Akt)—mammalian target of rapamycin (mTOR) signaling pathways (19-22). Approximately $15-20 \%$ of children with NF1 will develop an optic pathway tumor (pilocytic astrocytoma) (23-25). TSC is due to mutations in either the TSC1 gene or the TSC2 gene. TSC1 and TSC2 encode for the tumor suppressor proteins hamartin and tuberin respectively (26). The heterodimerization of hamartin 
with tuberin plays a role in the inhibition of the mTOR pathway (27). Subependymal giant cell astrocytomas (SEGAs) are low-grade tumors arising in the periventricular area. They are seen in about $5-20 \%$ of patients with TSC and may become symptomatic between the ages of 10 and 30 years $(28,29)$. A key phase I/II clinical trial completed by the Children's Hospital Medical Center in January 2014 investigated everolimus (RAD001), a mTOR kinase inhibitor, in patients 3 years of age and older with a definite diagnosis of TSC and an increasing SEGA tumor size (NCT00411619) (30). A 5-year post study analysis concluded that everolimus continued to demonstrate reduced SEGA tumor volumes while remaining to be well tolerated with no new safety concerns (31). In addition, a phase III study sponsored by Novartis Pharmaceuticals found that adjunctive treatment with everolimus significantly reduced seizure frequency compared to placebo in patients with TSC and treatment-resistant focal-onset seizures (NCT01713946) (32,33).

Previous clinical trials have also explored drugs that target MEK and BRAF for the treatment of PLGGs. A phase I/II study conducted by the Pediatric Brain Tumor Consortium (PBTC) found that selumetinib (AZD6244), a MEK1/2 inhibitor, was active in progressive, recurrent, or refractory pilocytic astrocytoma harboring BRAF alterations and NF1-assocaited PLGG (NCT01089101) (34). As a result of these promising findings, phase III studies comparing selumetinib to standard chemotherapy will proceed for newly diagnosed PLGGs in patients with and without NF1 (35). On the contrary, a phase II study of sorafenib (BRAF, VEGFR, PDGFR, KIT, RET, CRAF inhibitor) in children with recurrent or progressive low-grade astrocytoma was terminated early due to an unexpected high tumor progression rate (NCT01338857) (36). This clinical trial evaluated 11 patients, which included 3 patients with NF1 and 5 that tested positive for BRAF duplication. The rapid acceleration of tumor growth was irrespective of NF1 or tumor BRAF status. In vitro studies suggested that the effect may be related to paradoxical extracellular signalregulated kinase (ERK) activation. This finding emphasizes the importance of close monitoring for early tumor progression as more novel target agents become available for the treatment of PLGGs (37).

\section{Active molecular targeted clinical trials in PLGGs}

Clinical trials continue to investigate MEK and BRAF inhibitors for the treatment of PLGGs. St. Justine's
Hospital is studying the response rate to trametinib (MEK inhibitor) for children with LGGs and plexiform neurofibroma $(\mathrm{PN})$. In this phase I/II clinical trial, patients are placed into four groups: (I) patients with NF1 and progressing/refractory LGG, (II) patients with NF1 and PN, (III) patients with progressing/refractory LGG and a KIAA1549-BRAF fusion, and (IV) patients with progressing/refractory glioma with activation of the MAPK/ ERK pathway. This study also seeks to better understand the molecular mechanisms driving tumorigenesis and treatment resistance (NCT03363217) (38). Similarly, Novartis Pharmaceuticals sponsors phase I/II clinical trials of trametinib in combination with dabrafenib (BRAF inhibitor) in pediatric patients with tumors harboring BRAF $^{V 600}$ mutations (NCT02124772) (39), and in pediatric patients with $\mathrm{BRAF}^{\mathrm{V} 600 \mathrm{E}}$ mutated LGG and/or relapsed/ refractory HGG (NCT02684058) (40). Likewise, a phase I/ II study by the Children's Hospital Los Angeles is studying the experimental drug MEK162 (MEK1/2 inhibitor) in children with Ras/Raf pathway activated tumors. This study hopes to determine if MEK162 turns off the Ras/Raf/ MAP pathway and whether or not MEK162 causes tumors to reduce in size or stop growing. The different types of tumors in this study include LGG characterized by a BRAF truncated fusion (KIAA1549 and similar translocations), LGG in patients with NF1, and other tumors involving the Ras/Raf/MAP pathway. Another goal of the study is to learn if specific DNA abnormalities in the tumors can help predict the likelihood it will respond to treatment with MEK162 (NCT02285439) (41).

TAK-580 and vemurafenib (BRAF ${ }^{\mathrm{V} 600 \mathrm{E}}$ inhibitor) are additional novel drugs being explored in active clinical trials for the treatment of PLGGs. TAK-580 (MLN2480) is a type II BRAF inhibitor that uniquely targets the BRAF/MEK complex (42). This experimental drug has not been approved by the U.S. Food and Drug Administration (FDA) as a treatment for any disease, and this is the first time it will be given to children. Hence, an active phase I clinical trial will study the safety of TAK-580 in children and adolescent patients in three experimental arms: (I) recurrent/progressive RAS/RAF/ MEK/ERK activated pathway central nervous system or solid tumors, (II) recurrent/progressive LGG with a BRAF fusion, and (III) NF1 patients with recurrent/progressive LGG (NCT03429803) (43). Although more common in PLGGs, BRAF ${ }^{\mathrm{V} 600 \mathrm{E}}$ mutations have also been identified in PHGGs (44). An early phase I clinical trial by the University of California San Francisco is determining the safety 
of vemurafenib in pediatric patients with histologically confirmed diagnosis of gliomas WHO grades I-IV that are positive for the BRAF ${ }^{\mathrm{V} 600 \mathrm{E}}$ mutation (NCT01748149) (45). The patients must have failed at least one prior therapy besides surgery (radiation or chemotherapy) prior to beginning treatment with vemurafenib.

\section{PHGG}

Although PHGGs share histological and pathological features with adult HGGs, there are significant differences in their molecular profiles and locations (46). The WHO classifies PHGGs as either grade III or grade IV and therefore represent malignant and often very aggressive tumors. Approximately one-half of PHGGs occur in the brainstem. Of these brainstem gliomas, diffuse intrinsic pontine glioma (DIPG), or diffuse midline glioma according to the WHO's new nomenclature, is the most common $(3,47,48)$. Comparable to adults, few children with HGGs achieve long-term survival despite receiving a variety of therapies (49). Outcomes for these patients have remained poor with 5 -year survival rates of approximately $15-35 \%$ (47). DIPG is highly infiltrative in nature, which prevents surgical resection from being a viable treatment option (50). Radiation therapy remains the standard of care and has been shown to effectively slow tumor growth, but the overwhelming majority of those affected will not live 2 years post-diagnosis (9). This dismal prognosis has made molecular characterization of PHGGs like DIPG a priority in discovering alternative therapeutic interventions. A landmark study by $\mathrm{Wu}$ et al. [2012] found that approximately $80 \%$ of DIPGs and $20 \%$ of non-brainstem PHGGs possess a lysine to methionine mutation at position 27 on the histone tail (K27M mutation) in the genes encoding the histone variants H3.3 (H3F3A) and H3.1 (HIST1H3B) (51). Although the $\mathrm{H} 3.3 \mathrm{~K} 27 \mathrm{M}$ mutation is shared among midline PHGGS, the H3.1K27M mutation is exclusive to DIPG, and both mutations are seldom found in adult and cortical pediatric gliomas of similar grade (9). The type of H3 K27M mutation is also indicative of survival time and age of patient; longer survival and lower median age are associated with $\mathrm{H} 3.1 \mathrm{~K} 27 \mathrm{M}$ mutation compared to H3.3 K27M mutation. Because of the poor prognosis in patients with $\mathrm{H} 3 \mathrm{~K} 27$-mutated tumors, the 2016 WHO classification revision defined diffuse midline glioma, H3 K27M-mutant (grade IV) as a distinct entity $(3,52)$.

\section{Active molecular targeted clinical trials in PHGGs}

Innovative treatment approaches for PHGGs are underway in clinical trials such as the experimental drug ONC201 and a novel H3.3K27M specific vaccine. Gliomas with the H3 K27M mutation commonly overexpress dopamine receptor D2 (DRD2) and suppress dopamine receptor D5 (DRD5), leading to enhanced sensitivity to DRD2 antagonism (53). ONC201 is an orally active, small molecule DRD2 antagonist. This drug has the ability to cross the blood-brain barrier and causes p53-independent apoptosis in tumor cells by means of combined stress response activation and Akt/ERK inactivation (54). A phase II study of ONC201 administered every three weeks in adult recurrent glioblastoma (GBM) patients demonstrated that it was well tolerated and showed an objective radiographic response in a patient with a H3 K27M-mutant GBM (54). Given that a substantial proportion of DIPGs $(80 \%)$ harbor the H3 K27M mutation, a phase I study of oral ONC201 is recruiting pediatric patients with newly diagnosed DIPG and recurrent/refractory H3 K27M-mutant gliomas. Notably, this multicenter, five arm study includes an arm to determine H3 K27M DNA levels and drug concentrations in the cerebrospinal fluid (CSF) of pediatric H3 K27Mmutant glioma patients (NCT03416530) (55). The Pacific Pediatric Neuro-Oncology Consortium (PNOC) is assessing the safety of a novel $\mathrm{H} 3.3 \mathrm{~K} 27 \mathrm{M}$ specific vaccine in children with newly-diagnosed DIPG and other gliomas. In this phase I study, a specific $\mathrm{H} 3.3 \mathrm{~K} 27 \mathrm{M}$ peptide vaccine combined with the tetanus toxoid peptide, along with poly-ICLC (immunostimulant), will be administered in human leukocyte antigens (HLA)-A2 positive children and young adults with H3.3K27M DIPGs and other gliomas. Primary outcome measures are adverse events related to treatment and overall survival at 12 months. A secondary outcome measure looks at the induction of the H3.3K27M epitope-specific cytotoxic $\mathrm{T}$ lymphocyte response in postvaccine peripheral mononuclear cells. Other outcome measures include assessing $\mathrm{H} 3.3 \mathrm{~K} 27 \mathrm{M}$ expression status and infiltration of $\mathrm{H} 3.3 \mathrm{~K} 27 \mathrm{M}$ specific $\mathrm{T}$ cells, and determining whether circulating tumor DNA sequences in the patient's blood serve as biomarkers of tumor burden, response to therapy, or development of drug resistance (NCT02960230) (56).

Panobinostat has shown promise for the treatment of DIPG in the pre-clinical setting $(57,58)$. Elevated levels of H3 K27 acetylation in DIPG are epigenetic alterations targeted by HDAC inhibitors (HDACis). HDACis are anti- 
proliferative agents that modulate acetylation by targeting histone deacetylases (59). Panobinostat (LBH589) is a potent pan-HDACi that induces hyperacetylation of histones and other intracellular proteins, allowing for the expression of otherwise repressed genes, the inhibition of cellular proliferation, and the induction of apoptosis in malignant cells (60). To further explore this, the PBTC is conducting a phase I clinical trial of oral panobinostat for the treatment of children with recurrent/progressive (stratum 1) and nonprogressed DIPG (stratum 2) (NCT02717455) (61). A more recent phase I/II clinical trial is investigating the side effects of a panobinostat nanoparticle formulation MTX110 given intratumorally by convection-enhanced delivery in children with newly diagnosed DIPG (NCT03566199) (62). In addition to panobinostat, other targeted therapies currently in clinical trials for the treatment of PHGGs include GDC-0084, fimepinostat, abemaciclib, and PLB1001. Amplifications of the PI3K/AKT/mTOR signaling pathway have been identified in approximately $50 \%$ of DIPGs (63). GDC-0084 is a small molecule inhibitor of the PI3K/AKT/ mTOR pathway that efficiently crosses the blood-brain barrier. Recently, a phase I study of GDC-0084 conducted by St. Jude Children's Research Hospital in newly diagnosed DIPG and other diffuse midline H3 K27Mmutant gliomas after radiation therapy has been completed and will now advance to phase II trials (NCT03696355) (64). A new targeted validation study will assess fimepinostat (CUDC-907), an inhibitor of PI3K and histone deacetylase (HDAC), in treating children and young adults with newly diagnosed DIPG, recurrent HGGs, and MB. The primary objective of this early phase I clinical trial is to confirm that fimepinostat is able to cross the blood-brain barrier (NCT03893487) (65). Emory University is conducting a phase I clinical trial to assess abemaciclib (LY2835219), an inhibitor of cyclin dependent-kinases 4 and 6 (CDK4/6), in children and young adults with newly diagnosed DIPG and recurrent/refractory solid tumors including malignant brain tumors (NCT02644460) (66). Additionally, a phase I study is administering PLB1001, a potent selective c-Met inhibitor, in patients with PTPRZ1-MET fusion gene positive recurrent HGG (NCT02978261) (67).

An increasing number of clinical trials are being initiated to better understand the molecular abnormalities associated with PHGGs. For instance, the Children's Research Institute is collecting specimens prospectively from pediatric patients with DIPG or other brainstem gliomas in order to identify the unique molecular abnormalities that lead to tumor formation. Specimen collection includes serum, CSF, urine, and brain tumor tissue during and/or at autopsy. The goal of the study is to identify key molecules as biomarkers, which can be used as targets to design and test novel and more effective treatments (NCT01106794) (68). In another study, the Pacific PNOC is assessing the clinical benefit of using molecular profiling to design individualized treatment plans in children and young adults with HGGs (excluding DIPG). In this pilot trial, a specialized tumor board recommends individual treatment plans based on each patient's tumor gene expression, whole-exome sequencing, targeted panel profile (UCSF 500 gene panel), and RNAsequencing (NCT03739372) (69).

\section{MB}

MB has historically been stratified by histological appearance into four entities: (I) classic, (II) desmoplastic/ nodular, (III) large cell-anaplastic, and (IV) MB with extensive nodularity. Recently, the classification of $\mathrm{MB}$ has included clinically-significant molecular profiling data leading to its stratification into four distinct molecular subgroups: (I) WNT-activated, (II) SHH-activated (SHHactivated TP53-mutant and SHH-activated TP53wildtype), (III) group 3, and (IV) group $4(5,6,70)$. These subgroups have proven to exhibit distinct clinical features in terms of prognosis, response to therapy, anatomic location, demographic groups affected, and even cell of origin (4). WNT-activated tumors constitute $\sim 10 \%$ of MBs and typically carry the best prognosis. The favorable outcome may indicate that de-escalation of therapy would be most appropriate in such patients compared with more malignant subtypes (71). SHH-activated tumors constitute $~ 30 \%$ of MBs, making up the majority of infant (0-3 years) and adult (>16 years) MB while rarely affecting children 3-16 years old (5). These tumors carry a good-to-intermediate prognosis that varies significantly when accounting for age in conjunction with molecular and histopathological factors. Notably, SHH-activated TP53-mutated MB have poor outcomes and account for a large proportion of treatment failures (6). Targeted therapy trials for non-WNT/nonSHH MB (group 3 and group 4) are not as extensive as those targeting the WNT-activated and SHH-activated groups given that less is known about their pathogenesis. Groups 3 and 4 share a few genomic features in common, such as OTX2 amplification, gain of chromosomes 7, $17 \mathrm{q}$ and 18 , and loss of chromosomes $8,11 \mathrm{p}$ and the $\mathrm{X}$ chromosome in female patients (70). Group 3 tumors 
constitutes $25 \%$ of MBs, affects infants and children while sparing adults, affects males more than females, and has the poorest prognosis of all MB subtypes, owing largely to $M Y C$ amplification (70). These tumors exhibit a high level of large cell anaplastic histology and are the most likely subgroup to present with metastatic dissemination (5). Group 4 tumors are the most common subtype, accounting for $35 \%$ of $\mathrm{MBs}$, and yet is the least understood. Group $4 \mathrm{MB}$ exhibit an intermediate prognosis similar to $\mathrm{SHH}$-activated $\mathrm{MB}$ and affect males more than females. Although they are found in all age groups, group $4 \mathrm{MB}$ constitute the majority of childhood MB tumors (5). The molecular characterization of group $4 \mathrm{MB}$ is lacking and no model has yet been developed. Hence, a need exists for the identification of new molecular biomarkers. Notably, a study by Remke et al. [2011] found that follistatin-like 5 (FSTL5) expression is a marker of poor prognosis in non-WNT/non-SHH MB (72).

Not surprisingly, many of the novel targeted therapies for SHH-activated $\mathrm{MB}$ observed in clinical trials are inhibitors of the SHH pathway. This pathway has many diverse functions, the most relevant of which include neural patterning and differentiation. It includes the signaling ligand sonic hedgehog (Shh), receptor Patched (PTCH), signal transducer smoothened (SMO), and transcription factors Gli1, Gli2, and Gli3. In the absence of Shh signaling, PTCH inhibits SMO whereas binding of Shh to PTCH triggers a signaling cascade resulting in transcription of various genes responsible for proliferation (73). When aberrant, the SHH pathway fails to differentiate cerebellar granule neural precursor cells, leaving them in an immature stem-like proliferative state that could result in neoplasia. SMO inhibitors, such as vismodegib and sonidegib, have become incorporated into clinical trials for $\mathrm{SHH}$-activated MB after their initial approval by the FDA to treat basal cell carcinoma $(5,73)$. Preclinical data in mice suggested that SMO inhibitors were highly potent against mouse models of SHH-activated MB; however, they could cause growth defects in bone and teeth (74-76). Gajjar et al. [2013] published results of a phase I study of vismodegib (GDC0449) in children with recurrent or refractory MB. One of the goals in this clinical trial was to monitor for bone toxicities in pediatric patients. They found that vismodegib was well tolerated in children, and no drug-related bone toxicity was documented. In addition, 1 of 3 patients with $\mathrm{SHH}$-activated MB showed antitumor activity, whereas no antitumor activity was seen in patients with the other $\mathrm{MB}$ subgroups (74). As a result, a phase II study is currently recruiting patients with newly diagnosed $M B$ to be stratified into separate treatment arms based on both molecular subtype and clinical risk (NCT01878617) (77). In this clinical trial, vismodegib is added to the therapy regimen, which is the first time a $\mathrm{SHH}$ pathway inhibitor is given as part of the up-front treatment approach. Notably, three children with $\mathrm{SHH}$-activated $\mathrm{MB}$ treated with vismodegib were reported to develop growth plate fusions. As a result, the study was amended to restrict vismodegib to skeletally mature patients (78). Similarly, Novartis Pharmaceuticals completed a phase II study that evaluated the safety and efficacy of sonidegib (LDE225) in pediatric and adult patients with SHH-activated relapsed MB (NCT01708174) (79). In this clinical trial, 60 pediatric patients (39 MB, 21 other) and 16 adult patients (all MB) were enrolled. Consistent with the safety profile of adults, sonidegib was well tolerated in pediatric patients. However, growth plate changes were observed in prepubertal pediatric patients (wrist cartilage closure, knee cartilage closure, and knee subchondral condensation within growth plate). Regarding the efficacy sonidegib, the study found that 5 of the $10 \mathrm{MB}$ patients with $\mathrm{SHH}$-activated $\mathrm{MB}$ demonstrated complete or partial responses (80).

\section{Active molecular targeted clinical trials in pediatric MBs}

In addition to SMO inhibitors, other molecular targets, such as protein kinase inhibitors, are being explored in the treatment of MB. One such clinical trial sponsored by St. Jude Children's Research Hospital targets checkpoint kinase 1 (CK1) which mediates cell cycle checkpoint control and is essential for DNA repair while playing a role in chemotherapy resistance. This phase I clinical trial explores prexasertib (LY2606368), a selective inhibitor of protein kinase CK1, in combination with chemotherapy (cyclophosphamide or gemcitabine) in children and adolescents with refractory or recurrent group 3, group 4, or SHH-activated MB. Study participants will be stratified by the biological characteristics of their tumor to one of two treatment strata: stratum $\mathrm{A}$ is the combination of prexasertib and cyclophosphamide and stratum B is the combination of prexasertib and gemcitabine (NCT04023669) (81). Another clinical study by the PBTC is currently testing the safety and tolerability of CX-4945 (silmitasertib sodium), a selective inhibitor of casein kinase II (CK2), in children with recurrent, progressive, or refractory $\mathrm{SHH}$-activated MB. CX-4945 selectively binds to and inhibits CK2, which may lead to an inhibition of cellular proliferation (NCT03904862) (82-84). 
The use of novel experimental drugs to target specific mutations and molecular targets within distinct $\mathrm{MB}$ subgroups as described above have become increasingly popular and shown promise in phase I and II studies. However, other clinical trials seek to modulate existing treatment paradigms to accomplish similar results. Specifically, tailoring radiation and/or conventional chemotherapeutic interventions within $\mathrm{MB}$ subgroups is currently being investigated. As mentioned earlier, a phase II study by St. Jude Children's Research Hospital will first stratify newly diagnosed MB based on both molecular subtype (WNT, SHH or non-WNT/non-SHH) and clinical risk (low, standard, intermediate, or high risk), then proceed to clinical and molecular risk-directed therapy. This treatment approach will be used to assess the following: (I) whether treating low-risk WNT-activated MB with lower dose of radiation and lower dose of cyclophosphamide results in the same survival rate as past St. Jude studies with fewer side effects, (II) assess whether adding targeted chemotherapy following standard chemotherapy benefited SHH-activated MB patients, (III) determine if adding new chemotherapeutics to the standard chemotherapy improves outcomes for high risk non-WNT/non-SHH tumors, and (IV) determine the cure rate for standard risk nonWNT/non-SHH tumors that received reduced doses of cyclophosphamide compared to patients from the past St. Jude study (NCT01878617) (77). Similarly, the Children's Oncology Group (COG) is conducting a phase II trial to determine how well reduced doses of craniospinal radiation therapy and chemotherapy work in treating younger patients with newly diagnosed WNT-activated MB. The primary objective is to estimate the progression-free survival of children ( $>3$ years of age) with WNT-activated average-risk $\mathrm{MB}$ using reduced craniospinal radiation [18 Gray (Gy)] with a target boost to the tumor bed of 36 Gy for a total of 54 Gy and reduced chemotherapy (no vincristine during radiotherapy and reduced-dose maintenance chemotherapy such as cisplatin, vincristine, and lomustine). Secondary objectives include determining if DNA methylation profiling will accurately classify WNT-activated MB and evaluating the cognitive, social, emotional, behavioral, and quality of life functioning in the children receiving reduced doses. This study will provide new insights on whether or not giving reduced therapy can effectively kill tumor cells while also diminishing the late side effects of treatment (NCT02724579) (85).

\section{Ependymoma}

According to the WHO classification, ependymal tumors consist of four histopathological subtypes: subependymoma (grade I), myxopapillary ependymoma (grade I), ependymoma (grade II), ependymoma, RELA fusionpositive (grade II or III), and anaplastic ependymoma (grade III) (3). Anatomically, ependymomas (EPNs) are classified as supratentorial (ST), posterior fossa (PF), or spinal (SP). Standard treatment of EPNs consists of maximal safe surgical resection followed by radiation to the primary tumor site. Unfortunately, even after gross total resection and treatment with adjuvant radiotherapy and/or chemotherapy, children with EPNs have poor long-term survival (86). It has recently been discovered that ST-EPNs and PF-EPNs have distinct molecular profiles and are likely to be different diseases (87). Briefly, ST-EPNs may be further classified as either C11orf95-RELA fusion-positive (ST-EPN-RELA) (70\%) or YAP1 fusion-positive (ST-EPNYAP1), and PF-EPNs can be broken into two molecular subgroups based on methylation patterns of $\mathrm{CpG}$ islands: PF-EPN-A which occurs in younger patients and carries a worse prognosis in terms of progression-free and overall survival, and PF-EPN-B which occurs in older patients and carries a better prognosis. Given the difference in prognosis between PF-EPN-A and PF-EPN-B, some have suggested treating PF-EPN-B patients with surgery alone, although no clinical trials have demonstrated this. Furthermore, no driver mutations have yet been isolated in either PF-EPN subgroup (88). Despite the recent significant advances in understanding the molecular profiles of EPNs, there are currently no active clinical trials treating these tumors using molecular stratification. However, EPNs may be included in studies which encompass multiple pediatric brain tumor types such as SJDAWN and National Cancer Institute (NCI)-COG Pediatric MATCH trials described below.

\section{Molecular targeted clinical trials encompassing multiple pediatric brain tumors}

SJDAWN is a recently launched study by St. Jude Children's Research Hospital that evaluates molecularlydriven doublet therapies for children and young adults with recurrent brain tumors, including MBs, EPNs, and HGGs. This unique phase I clinical trial uses two combined novel drugs (a doublet) based on tumor type and molecular characteristics, and study participants will receive 
one of three drug combinations: (I) ribociclib (CDK4/6 inhibitor) and gemcitabine, (II) ribociclib and trametinib, or (III) ribociclib and sonidegib (NCT03434262) (89). Similarly, the NCI-COG Pediatric MATCH (Molecular Analysis for Therapy Choice) is a nationwide clinical trial that includes various types of solid tumors, including progressed or recurrent brain tumors. This study utilizes experimental targeted drugs based on genetic alterations found in the tumor, regardless of the tumor type. Each treatment arm tests an experimental drug that has shown activity against tumors with specific genetic changes, such as selumetinib and vemurafenib, which target MEK and BRAF respectively, both of which have shown promise for the targeted treatment of pediatric brain tumors (90).

\section{Conclusions}

Significant progress has been made in the understanding of pediatric brain tumors through recent advances in molecular technologies. This new knowledge has resulted in targeted treatments currently being explored in a variety of active clinical trials. For example, the promising experimental drug selumetinib for the treatment of PLGGs, the development of a novel H3.3K27M specific vaccine for DIPG and other gliomas, and risk-stratified therapy for children with WNT-activated subgroup MB, which has a very good prognosis and may benefit from reduced radiotherapy and chemotherapy potentially decreasing long-term side effects. However, a great need persists for the development of clinical trials based on molecular stratification for children with very high risk brain tumors that carry a poor prognosis such as SHH-activated TP53 mutated $\mathrm{MB}$, group $3 \mathrm{MB}$, and $\mathrm{H} 3 \mathrm{~K} 27 \mathrm{M}$-mutant diffuse midline gliomas. Furthermore, with brain tumors as the leading cause of cancer death in children and adolescents, ongoing clinical trials, such as the ones mentioned in this review, are critical in the advancement of new therapeutic options to improve patient outcomes and reduce long-term side effects.

\section{Acknowledgments}

Funding: None.

\section{Footnote}

Conflicts of Interest: All authors have completed the ICMJE uniform disclosure form (available at http://dx.doi. org/10.21037/tp.2020.03.04). The authors have no conflicts of interest to declare.

Ethical Statement: The authors are accountable for all aspects of the work in ensuring that questions related to the accuracy or integrity of any part of the work are appropriately investigated and resolved.

Open Access Statement: This is an Open Access article distributed in accordance with the Creative Commons Attribution-NonCommercial-NoDerivs 4.0 International License (CC BY-NC-ND 4.0), which permits the noncommercial replication and distribution of the article with the strict proviso that no changes or edits are made and the original work is properly cited (including links to both the formal publication through the relevant DOI and the license). See: https://creativecommons.org/licenses/by-nc-nd/4.0/.

\section{References}

1. Curtin SC, Minino AM, Anderson RN. Declines in Cancer Death Rates Among Children and Adolescents in the United States, 1999-2014. NCHS Data Brief 2016;(257):1-8.

2. Turner CD, Rey-Casserly C, Liptak CC, et al. Late effects of therapy for pediatric brain tumor survivors. J Child Neurol 2009;24:1455-63.

3. Louis DN, Perry A, Reifenberger G, et al. The 2016 World Health Organization Classification of Tumors of the Central Nervous System: a summary. Acta Neuropathol 2016;131:803-20.

4. Northcott PA, Korshunov A, Witt H, et al. Medulloblastoma comprises four distinct molecular variants. J Clin Oncol 2011;29:1408-14.

5. Taylor MD, Northcott PA, Korshunov A, et al. Molecular subgroups of medulloblastoma: the current consensus. Acta Neuropathol 2012;123:465-72.

6. Zhukova N, Ramaswamy V, Remke M, et al. Subgroupspecific prognostic implications of TP53 mutation in medulloblastoma. J Clin Oncol 2013;31:2927-35.

7. Horbinski C, Hamilton RL, Nikiforov Y, et al. Association of molecular alterations, including BRAF, with biology and outcome in pilocytic astrocytomas. Acta Neuropathol 2010;119:641-9.

8. Venneti S, Huse JT. The evolving molecular genetics of low-grade glioma. Adv Anat Pathol 2015;22:94-101.

9. Morales La Madrid A, Hashizume R, Kieran MW. Future Clinical Trials in DIPG: Bringing Epigenetics to the Clinic. Front Oncol 2015;5:148. 
10. Ostrom QT, Gittleman H, Fulop J, et al. CBTRUS Statistical Report: Primary Brain and Central Nervous System Tumors Diagnosed in the United States in 20082012. Neuro Oncol 2015;17 Suppl 4:iv1-62.

11. Sievert AJ, Fisher MJ. Pediatric low-grade gliomas. J Child Neurol 2009;24:1397-408.

12. Lin A, Rodriguez FJ, Karajannis MA, et al. BRAF alterations in primary glial and glioneuronal neoplasms of the central nervous system with identification of 2 novel KIAA1549:BRAF fusion variants. J Neuropathol Exp Neurol 2012;71:66-72.

13. Garnett MJ, Marais R. Guilty as charged: B-RAF is a human oncogene. Cancer Cell 2004;6:313-9.

14. Sievert AJ, Jackson EM, Gai X, et al. Duplication of $7 q 34$ in pediatric low-grade astrocytomas detected by highdensity single-nucleotide polymorphism-based genotype arrays results in a novel BRAF fusion gene. Brain Pathol 2009;19:449-58.

15. Pfister S, Janzarik WG, Remke M, et al. BRAF gene duplication constitutes a mechanism of MAPK pathway activation in low-grade astrocytomas. J Clin Invest 2008;118:1739-49.

16. Jones DT, Kocialkowski S, Liu L, et al. Oncogenic RAF1 rearrangement and a novel BRAF mutation as alternatives to KIAA1549:BRAF fusion in activating the MAPK pathway in pilocytic astrocytoma. Oncogene 2009;28:2119-23.

17. Penman CL, Faulkner C, Lowis SP, et al. Current Understanding of BRAF Alterations in Diagnosis, Prognosis, and Therapeutic Targeting in Pediatric LowGrade Gliomas. Front Oncol 2015;5:54.

18. Lassaletta A, Zapotocky M, Mistry M, et al. Therapeutic and Prognostic Implications of BRAF V600E in Pediatric Low-Grade Gliomas. J Clin Oncol 2017;35:2934-41.

19. Sandsmark DK, Zhang H, Hegedus B, et al. Nucleophosmin mediates mammalian target of rapamycindependent actin cytoskeleton dynamics and proliferation in neurofibromin-deficient astrocytes. Cancer Res 2007;67:4790-9.

20. Banerjee S, Crouse NR, Emnett RJ, et al. Neurofibromatosis-1 regulates mTOR-mediated astrocyte growth and glioma formation in a TSC/Rheb-independent manner. Proc Natl Acad Sci U S A 2011;108:15996-6001.

21. Johannessen CM, Reczek EE, James MF, et al. The NF1 tumor suppressor critically regulates TSC2 and mTOR. Proc Natl Acad Sci U S A 2005;102:8573-8.

22. Watson AL, Anderson LK, Greeley AD, et al. Cotargeting the MAPK and PI3K/AKT/mTOR pathways in two genetically engineered mouse models of schwann cell tumors reduces tumor grade and multiplicity. Oncotarget 2014;5:1502-14.

23. Listernick R, Charrow J, Greenwald M, et al. Natural history of optic pathway tumors in children with neurofibromatosis type 1: a longitudinal study. J Pediatr 1994;125:63-6.

24. Lewis RA, Gerson LP, Axelson KA, et al. von Recklinghausen neurofibromatosis. II. Incidence of optic gliomata. Ophthalmology 1984;91:929-35.

25. Gutmann DH, Rasmussen SA, Wolkenstein P, et al. Gliomas presenting after age 10 in individuals with neurofibromatosis type 1 (NF1). Neurology 2002;59:759-61.

26. Lamb RF, Roy C, Diefenbach TJ, et al. The TSC1 tumour suppressor hamartin regulates cell adhesion through ERM proteins and the GTPase Rho. Nat Cell Biol 2000;2:281-7.

27. Kwiatkowski DJ, Manning BD. Molecular basis of giant cells in tuberous sclerosis complex. N Engl J Med 2014;371:778-80.

28. Walls GV, Reed AA, Jeyabalan J, et al. Proliferation rates of multiple endocrine neoplasia type 1 (MEN1)-associated tumors. Endocrinology 2012;153:5167-79.

29. Goh S, Butler W, Thiele EA. Subependymal giant cell tumors in tuberous sclerosis complex. Neurology 2004;63:1457-61.

30. ClinicalTrials.gov. Bethesda (MD): National Library of Medicine (US). 2000 Feb 29 - Identifier NCT00411619, Everolimus (RAD001) Therapy of Giant Cell Astrocytoma in Patients With Tuberous Sclerosis Complex; 2006 Dec 14. Available online: https://clinicaltrials.gov/ct2/show/ NCT00411619? term=NCT00411619\&draw=2\&rank=1.

31. Franz DN, Agricola K, Mays M, et al. Everolimus for subependymal giant cell astrocytoma: 5-year final analysis. Ann Neurol 2015;78:929-38.

32. ClinicalTrials.gov. Bethesda (MD): National Library of Medicine (US). 2000 Feb 29 - Identifier NCT01713946, A Placebo-controlled Study of Efficacy \& Safety of 2 Trough-ranges of Everolimus as Adjunctive Therapy in Patients With Tuberous Sclerosis Complex (TSC) \& Refractory Partial-onset Seizures (EXIST-3); 2012 Oct 25. Available online: https://clinicaltrials.gov/ct2/show/NCT0 3416530? term=NCT03416530\&draw=2\&rank=1

33. French JA, Lawson JA, Yapici Z, et al. Adjunctive everolimus therapy for treatment-resistant focal-onset seizures associated with tuberous sclerosis (EXIST-3): a phase 3, randomised, double-blind, placebo-controlled 
study. Lancet 2016;388:2153-63.

34. ClinicalTrials.gov. Bethesda (MD): National Library of Medicine (US). 2000 Feb 29 - Identifier NCT01089101, Selumetinib in Treating Young Patients With Recurrent or Refractory Low Grade Glioma; 2010 Mar 18. Available online: https://clinicaltrials.gov/ct2/show/NCT01089101? term=NCT01089101\&draw=2\&rank=1

35. Fangusaro J, Onar-Thomas A, Young Poussaint T, et al. Selumetinib in paediatric patients with BRAF-aberrant or neurofibromatosis type 1-associated recurrent, refractory, or progressive low-grade glioma: a multicentre, phase 2 trial. Lancet Oncol 2019;20:1011-22.

36. ClinicalTrials.gov. Bethesda (MD): National Library of Medicine (US). 2000 Feb 29 - Identifier NCT01338857, Sorafenib in Children and Young Adults With Recurrent or Progressive Low-Grade Astrocytomas; 2011 Apr 20. Available online: https://clinicaltrials.gov/ct2/show/NCT0 1338857 ? term=NCT01338857\&draw=2\&rank=1

37. Karajannis MA, Legault G, Fisher MJ, et al. Phase II study of sorafenib in children with recurrent or progressive lowgrade astrocytomas. Neuro Oncol 2014;16:1408-16.

38. ClinicalTrials.gov. Bethesda (MD): National Library of Medicine (US). 2000 Feb 29 - Identifier NCT03363217, Trametinib for Pediatric Neuro-oncology Patients With Refractory Tumor and Activation of the MAPK/ ERK Pathway; 2017 Dec 6. Available online: https:// clinicaltrials.gov/ct2/show/NCT03363217? term=NCT03 $363217 \&$ draw $=2 \&$ rank $=1$

39. ClinicalTrials.gov. Bethesda (MD): National Library of Medicine (US). 2000 Feb 29 - Identifier NCT02124772, Study to Investigate Safety, Pharmacokinetic (PK), Pharmacodynamic (PD) and Clinical Activity of Trametinib in Subjects With Cancer or Plexiform Neurofibromas and Trametinib in Combination With Dabrafenib in Subjects With Cancers Harboring V600 Mutations; 2014 Apr 28. Available online: https://clinicaltrials.gov/ct2/show/NCT0 2124772 ? term=NCT02 124772 \&draw=2\&rank=1

40. ClinicalTrials.gov. Bethesda (MD): National Library of Medicine (US). 2000 Feb 29 - Identifier NCT02684058, Phase II Pediatric Study With Dabrafenib in Combination With Trametinib in Patients With HGG and LGG; 2016 Feb 17. Available online: https:// clinicaltrials.gov/ct2/show/NCT02684058? term=NCT0 2684058\&draw=2\&rank=1

41. ClinicalTrials.gov. Bethesda (MD): National Library of Medicine (US). 2000 Feb 29 - Identifier NCT02285439, Phase I/II Study of MEK162 for Children With Ras/Raf Pathway Activated Tumors; 2014 Nov 7. Available online:
https://clinicaltrials.gov/ct2/show/NCT02285439? term= NCT02285439\&draw $=2 \&$ rank $=1$

42. Wright KD, Zimmerman MA, Fine E, et al. Kieran, and Susan Chi. LGG-26. Type II BRAF inhibitor TAK-580 shows promise for upcoming clinal trial as evidenced by single patient IND study [abstract]. Neuro-Oncology 2018;20:i110.

43. ClinicalTrials.gov. Bethesda (MD): National Library of Medicine (US). 2000 Feb 29 - Identifier NCT03429803, TAK-580 In Gliomas and Other Tumors; 2018 Feb 12. Available online: https://clinicaltrials.gov/ct2/show/NCT0 3429803 ?term $=$ NCT03429803 \& draw $=2 \&$ rank $=1$

44. Dahiya S, Emnett RJ, Haydon DH, et al. BRAF-V600E mutation in pediatric and adult glioblastoma. Neuro Oncol 2014;16:318-9.

45. ClinicalTrials.gov. Bethesda (MD): National Library of Medicine (US). 2000 Feb 29 - Identifier NCT01748149, Vemurafenib in Children With Recurrent/Refractory BRAF Gene V600E (BRAFV600E)-Mutant Gliomas; 2012 Dec 12. Available online: https://clinicaltrials.gov/ct2/show/NC T01748149? term=NCT01748149\&draw=2\&rank=1

46. Wu G, Diaz AK, Paugh BS, et al. The genomic landscape of diffuse intrinsic pontine glioma and pediatric nonbrainstem high-grade glioma. Nat Genet 2014;46:444-50.

47. Broniscer A, Gajjar A. Supratentorial high-grade astrocytoma and diffuse brainstem glioma: two challenges for the pediatric oncologist. Oncologist 2004;9:197-206.

48. Buczkowicz P, Hawkins C. Pathology, Molecular Genetics, and Epigenetics of Diffuse Intrinsic Pontine Glioma. Front Oncol 2015;5:147.

49. Fangusaro J. Pediatric high grade glioma: a review and update on tumor clinical characteristics and biology. Front Oncol 2012;2:105.

50. Kambhampati M, Perez JP, Yadavilli S, et al. A standardized autopsy procurement allows for the comprehensive study of DIPG biology. Oncotarget 2015;6:12740-7.

51. Wu G, Broniscer A, McEachron TA, et al. Somatic histone $\mathrm{H} 3$ alterations in pediatric diffuse intrinsic pontine gliomas and non-brainstem glioblastomas. Nat Genet 2012;44:251-3.

52. Castel D, Philippe C, Calmon R, et al. Histone H3F3A and HIST1H3B K27M mutations define two subgroups of diffuse intrinsic pontine gliomas with different prognosis and phenotypes. Acta Neuropathol 2015;130:815-27.

53. Hall MD, Odia Y, Allen JE, et al. First clinical experience with DRD2/3 antagonist ONC201 in H3 K27M-mutant pediatric diffuse intrinsic pontine glioma: a case report. J Neurosurg Pediatr 2019;5:1-7. 
54. Arrillaga-Romany I, Chi AS, Allen JE, et al. A phase 2 study of the first imipridone ONC201, a selective DRD2 antagonist for oncology, administered every three weeks in recurrent glioblastoma. Oncotarget 2017;8:79298-304.

55. ClinicalTrials.gov. Bethesda (MD): National Library of Medicine (US). 2000 Feb 29 - Identifier NCT03416530, ONC201 in Pediatric H3 K27M Gliomas; 2018 Jan 31. Available online: https://clinicaltrials.gov/ct2/show/NCT0 3416530? term=NCT03416530\&draw=2\&rank=1

56. ClinicalTrials.gov. Bethesda (MD): National Library of Medicine (US). 2000 Feb 29 - Identifier NCT02960230, H3.3K27M Peptide Vaccine for Children With Newly Diagnosed DIPG and Other Gliomas; 2016 Nov 9. Available online: https://clinicaltrials.gov/ct2/show/NCT0 2960230? term =NCT02960230\&draw=2\&rank=1

57. Grasso CS, Tang Y, Truffaux N, et al. Functionally defined therapeutic targets in diffuse intrinsic pontine glioma. Nat Med 2015;21:827.

58. Hennika T, Hu G, Olaciregui NG, et al. Pre-Clinical Study of Panobinostat in Xenograft and Genetically Engineered Murine Diffuse Intrinsic Pontine Glioma Models. PLoS One 2017;12:e0169485.

59. New M, Olzscha H, La Thangue NB. HDAC inhibitorbased therapies: can we interpret the code? Mol Oncol 2012;6:637-56.

60. Anne M, Sammartino D, Barginear MF, et al. Profile of panobinostat and its potential for treatment in solid tumors: an update. Onco Targets Ther 2013;6:1613-24.

61. ClinicalTrials.gov. Bethesda (MD): National Library of Medicine (US). 2000 Feb 29 - Identifier NCT02717455, Trial of Panobinostat in Children With Diffuse Intrinsic Pontine Glioma (PBTC-047); 2016 Mar 23. Available online: https://clinicaltrials.gov/ct2/show/NCT02717455? term=NCT02717455\&draw=2\&rank=1

62. ClinicalTrials.gov. Bethesda (MD): National Library of Medicine (US). 2000 Feb 29 - Identifier NCT03566199, MTX110 by Convection-Enhanced Delivery in Treating Participants With Newly-Diagnosed Diffuse Intrinsic Pontine Glioma (PNOC015); 2018 Jun 25. Available online: https://clinicaltrials.gov/ct2/show/NCT03566199? term=NCT03566199\&draw=2\&rank=1

63. Paugh BS, Broniscer A, Qu C, et al. Genome-wide analyses identify recurrent amplifications of receptor tyrosine kinases and cell-cycle regulatory genes in diffuse intrinsic pontine glioma. J Clin Oncol 2011;29:3999-4006.

64. ClinicalTrials.gov. Bethesda (MD): National Library of Medicine (US). 2000 Feb 29 - Identifier NCT03696355, Study of GDC-0084 in Pediatric Patients With Newly
Diagnosed Diffuse Intrinsic Pontine Glioma or Diffuse Midline Gliomas; 2018 Oct 4. Available online: https:// clinicaltrials.gov/ct2/show/NCT03696355?term=NCT03 696355 \&draw $=2 \&$ rank $=1$

65. ClinicalTrials.gov. Bethesda (MD): National Library of Medicine (US). 2000 Feb 29 - Identifier NCT03893487, Fimepinostat in Treating Brain Tumors in Children and Young Adults (PNOC016); 2019 Mar 28. Available online: https:/clinicaltrials.gov/ct2/show/NCT03893487?term= NCT03893487\&draw=2\&rank=1

66. ClinicalTrials.gov. Bethesda (MD): National Library of Medicine (US). 2000 Feb 29 - Identifier NCT02644460, Abemaciclib in Children With DIPG or Recurrent/ Refractory Solid Tumors (AflacST1501); 2015 Dec 31. Available online: https://clinicaltrials.gov/ct2/show/NCT0 2644460? term=NCT02644460 \& draw=2 \& rank=1

67. ClinicalTrials.gov. Bethesda (MD): National Library of Medicine (US). 2000 Feb 29 - Identifier NCT02978261, Study of a c-Met Inhibitor PLB1001 in Patients With PTPRZ1-MET Fusion Gene Positive Recurrent Highgrade Gliomas; 2016 Nov 30. Available online: https:// clinicaltrials.gov/ct2/show/NCT02978261? term=NCT02 978261\&draw $=2 \&$ rank $=1$

68. ClinicalTrials.gov. Bethesda (MD): National Library of Medicine (US). 2000 Feb 29 - Identifier NCT01106794, Molecular Analysis of Samples From Patients With Diffuse Intrinsic Pontine Glioma and Brainstem Glioma; 2010 Apr 20. Available online: https://clinicaltrials.gov/ ct2/show/NCT01106794? term $=$ NCT01 106794\&draw $=2$ \&rank=1

69. ClinicalTrials.gov. Bethesda (MD): National Library of Medicine (US). 2000 Feb 29 - Identifier NCT03739372, Clinical Benefit of Using Molecular Profiling to Determine an Individualized Treatment Plan for Patients With High Grade Glioma (PNOC008); 2018 Nov 12. Available online: https://clinicaltrials.gov/ct2/show/NCT0 3739372 ? term =NCT03739372\&draw $=2 \&$ rank $=1$

70. Northcott PA, Dubuc AM, Pfister S, et al. Molecular subgroups of medulloblastoma. Expert Rev Neurother 2012;12:871-84.

71. Ramaswamy V, Northcott PA, Taylor MD. FISH and chips: the recipe for improved prognostication and outcomes for children with medulloblastoma. Cancer Genet 2011;204:577-88.

72. Remke M, Hielscher T, Korshunov A, et al. FSTL5 is a marker of poor prognosis in non-WNT/non-SHH medulloblastoma. J Clin Oncol 2011;29:3852-61.

73. Jain S, Song R, Xie J. Sonidegib: mechanism of action, 
pharmacology, and clinical utility for advanced basal cell carcinomas. Onco Targets Ther 2017;10:1645-53.

74. Gajjar A, Stewart CF, Ellison DW, et al. Phase I study of vismodegib in children with recurrent or refractory medulloblastoma: a pediatric brain tumor consortium study. Clin Cancer Res 2013;19:6305-12.

75. Kimura H, Ng JM, Curran T. Transient inhibition of the Hedgehog pathway in young mice causes permanent defects in bone structure. Cancer Cell 2008;13:249-60.

76. Seidel K, Ahn CP, Lyons D, et al. Hedgehog signaling regulates the generation of ameloblast progenitors in the continuously growing mouse incisor. Development 2010;137:3753-61.

77. ClinicalTrials.gov. Bethesda (MD): National Library of Medicine (US). 2000 Feb 29 - Identifier NCT01878617, A Clinical and Molecular Risk-Directed Therapy for Newly Diagnosed Medulloblastoma; 2013 Jun 17. Available online: https://clinicaltrials.gov/ct2/show/NCT01878617

78. Robinson GW, Kaste SC, Chemaitilly W, et al. Irreversible growth plate fusions in children with medulloblastoma treated with a targeted hedgehog pathway inhibitor. Oncotarget 2017;8:69295-302.

79. ClinicalTrials.gov. Bethesda (MD): National Library of Medicine (US). 2000 Feb 29 - Identifier NCT01708174, A Phase II Study of Oral LDE225 in Patients With HedgeHog (Hh)-Pathway Activated Relapsed Medulloblastoma (MB); 2012 Oct 16. Available online: https://clinicaltrials. gov/ct2/show/NCT01708174?term=NCT01708174\&dra $\mathrm{w}=2 \& \operatorname{rank}=1$

80. Kieran MW, Chisholm J, Casanova M, et al. Phase I study of oral sonidegib (LDE225) in pediatric brain and solid tumors and a phase II study in children and adults with relapsed medulloblastoma. Neuro Oncol 2017;19:1542-52.

81. ClinicalTrials.gov. Bethesda (MD): National Library of Medicine (US). 2000 Feb 29 - Identifier NCT04023669, Evaluation of LY2606368 Therapy in Combination With Cyclophosphamide or Gemcitabine for Children and Adolescents With Refractory or Recurrent Group 3/ Group 4 or SHH Medulloblastoma Brain Tumors; 2019 Jul 17. Available online: https://clinicaltrials.gov/ct2/show/ NCT04023669? term=NCT04023669\&draw=2\&rank=1

82. Siddiqui-Jain A, Drygin D, Streiner N, et al. CX-4945, an orally bioavailable selective inhibitor of protein kinase CK2, inhibits prosurvival and angiogenic signaling and exhibits antitumor efficacy. Cancer Res 2010;70:10288-98.

83. Ku MJ, Park JW, Ryu BJ, et al. CK2 inhibitor CX4945 induces sequential inactivation of proteins in the signaling pathways related with cell migration and suppresses metastasis of A549 human lung cancer cells. Bioorg Med Chem Lett 2013;23:5609-13.

84. ClinicalTrials.gov. Bethesda (MD): National Library of Medicine (US). 2000 Feb 29 - Identifier NCT03904862, Testing the Safety and Tolerability of CX-4945 in Patients With Recurrent Medulloblastoma Who May or May Not Have Surgery; 2019 Apr 5. Available online: https:// clinicaltrials.gov/ct2/show/NCT03904862? term=NCT03 $904862 \&$ draw $=2 \&$ rank $=1$

85. ClinicalTrials.gov. Bethesda (MD): National Library of Medicine (US). 2000 Feb 29 - Identifier NCT02724579, Reduced Craniospinal Radiation Therapy and Chemotherapy in Treating Younger Patients With Newly Diagnosed WNT-Driven Medulloblastoma; 2016 Mar 31. Available online: https://clinicaltrials.gov/ct2/show/NCT0 2724579? term=NCT02724579\&draw=2\&rank=1

86. Marinoff AE, Ma C, Guo D, et al. Rethinking childhood ependymoma: a retrospective, multi-center analysis reveals poor long-term overall survival. J Neurooncol 2017;135:201-11.

87. Fukuoka K, Kanemura Y, Shofuda T, et al. Significance of molecular classification of ependymomas: C11orf95RELA fusion-negative supratentorial ependymomas are a heterogeneous group of tumors. Acta Neuropathol Commun 2018;6:134.

88. Ramaswamy V, Hielscher T, Mack SC, et al. Therapeutic Impact of Cytoreductive Surgery and Irradiation of Posterior Fossa Ependymoma in the Molecular Era: A Retrospective Multicohort Analysis. J Clin Oncol 2016;34:2468-77.

89. ClinicalTrials.gov. Bethesda (MD): National Library of Medicine (US). 2000 Feb 29 - Identifier NCT03434262, SJDAWN: St. Jude Children's Research Hospital Phase 1 Study Evaluating Molecularly-Driven Doublet Therapies for Children and Young Adults With Recurrent Brain Tumors; 2018 Feb 15. Available online: https:// clinicaltrials.gov/ct2/show/NCT03434262? term=NCT03 $434262 \&$ draw $=2 \&$ rank $=1$

90. NCI-COG. National Cancer Institute-Children's Oncology Group Pediatric MATCH 2018, October 2. Available online: https:/www.cancer.gov/about-cancer/ treatment/clinical-trials/nci-supported/pediatric-match

Cite this article as: Hanz SZ, Adeuyan O, Lieberman G, Hennika T. Clinical trials using molecular stratification of pediatric brain tumors. Transl Pediatr 2020;9(2):144-156. doi: 10.21037/tp.2020.03.04 